\title{
Investigating the potential genetic association between RANBP9 polymorphisms and the risk of schizophrenia
}

\author{
JOON SEOL BAE ${ }^{1 *}$, JASON YONGHA KIM ${ }^{2 *}$, BYUNG-LAE PARK ${ }^{3}$, HYUN SUB CHEONG $^{3}$, JEONG-HYUN KIM ${ }^{2}$, \\ SUHG NAMGOONG ${ }^{2,3}$, JI-ON KIM ${ }^{2,3}$, CHUL SOO PARK ${ }^{4}$, BONG-JO KIM ${ }^{4}$, CHEOL-SOON LEE ${ }^{4}$, MIGYUNG LEE $^{5}$, \\ WOO HYUK CHOI ${ }^{6}$, TAE-MIN SHIN ${ }^{6}$, JAEUK HWANG $^{7}$, HYOUNG DOO SHIN $^{2,3}$ and SUNG-IL WOO ${ }^{7}$ \\ ${ }^{1}$ Laboratory of Translational Genomics, Samsung Genome Institute, Samsung Medical Center, Seoul 135-710; \\ ${ }^{2}$ Laboratory of Genomic Diversity, Department of Life Science, Sogang University, Seoul 121-742; \\ ${ }^{3}$ Department of Genetic Epidemiology, SNP Genetics, Inc., Sogang University, Seoul 121-742; \\ ${ }^{4}$ Department of Psychiatry, College of Medicine, Gyeongsang National University, Jinju, Gyeongsang Nam Do, 660-751; \\ ${ }^{5}$ Department of General Psychiatry, Seoul National Hospital, Seoul 110-799; ${ }^{6}$ Department of Biomedical Engineering, \\ Yonsei University, Wonju, Gangwon 220-710; ${ }^{7}$ Department of Neuropsychiatry, Soonchunhyang University Hospital, \\ Seoul 420-767, Republic of Korea
}

Received February 11, 2014; Accepted November 5, 2014

DOI: $10.3892 / \mathrm{mmr} .2014 .3045$

\begin{abstract}
Schizophrenia is a serious mental disorder that is affected by genetic and environmental factors. As the disease has a high heritability rate, genetic studies identifying candidate genes for schizophrenia have been conducted in various populations. The gene for human Ran-binding protein 9 (RANBP9) is a newly discovered candidate gene for schizophrenia. As RANBP9 is a small guanosine-5'-triphosphate-binding protein that interacts with the disrupted in schizophrenia 1 protein, it is considered to be an important molecule in the pathogenesis of schizophrenia. However, to date, no study has examined the possible association between the genetic variations of RANBP9 and the risk of schizophrenia. In the present study, it was hypothesized that RANBP9 variations may influence the risk of schizophrenia. In order to investigate the association between RANBP9 polymorphisms and the risk of schizophrenia and smooth pursuit eye movement (SPEM) abnormalities, a case-control association analysis was performed. Using a
\end{abstract}

Correspondence to: Professor Sung-Il Woo, Department of Neuropsychiatry, Soonchunhyang University Hospital, 59 Daesagwan-ro, Yongsan-gu, Seoul 420-767, Republic of Korea E-mail: siwoo@hosp.sch.ac.kr

Professor Hyoung Doo Shin, Laboratory of Genomic Diversity, Department of Life Science, Sogang University, 35 Baekbeom-ro, Mapo-gu, Seoul 121-742, Republic of Korea

E-mail: hdshin@sogang.ac.kr

*Contributed equally

Key words: Ran binding protein 9, single-nucleotide polymorphism, smooth pursuit eye movement abnormality, haplotype, schizophrenia
TaqMan assay, five single-nucleotide polymorphisms and an insertion/deletion variation within the start codon region of RANBP9 were genotyped. Five major haplotypes were identified in 449 patients with schizophrenia and 393 unrelated healthy individuals as controls (total, $\mathrm{n}=842$ ). However, the association analyses revealed no associations between all genetic variants and schizophrenia and SPEM abnormality. To the best of our knowledge, this is the first study to investigate an association between RANBP9 polymorphisms and schizophrenia and SPEM abnormality. The findings of allele frequencies and association results in this study may aid in further genetic etiological studies in schizophrenia in various populations.

\section{Introduction}

Schizophrenia is a severe cognitive disorder that is characterized by psychosis, disorganized speech and behavioral symptoms. The observed lifetime prevalence estimates and morbidity rate of this condition in the general population is 4.0 in 1,000 and 7.2 in 1,000 people, respectively $(1,2)$. In the etiology of schizophrenia, the disease development is attributable to a combination of environmental and genetic risk factors. Previous molecular genetic studies have identified abnormalities in the structure of oligodendrocytes and myelin in schizophrenic brains (3-8), factors that may contribute to the disorganized behavioral symptoms in schizophrenia patients (9). Despite the well-defined factors involved in disease development, the exact molecular mechanisms of schizophrenia remain unclear and require further investigation.

Proteins involved in the dopamine or glutamate signaling pathways, which regulate nerve growth and development, have been hypothesized to be candidate molecules for indicating the risk of schizophrenia. The human Ran-binding protein 9 (RANBP9; NM_005493.2) is a member of the Ran-binding family of proteins and interacts with multiple receptors, acting as a scaffold protein for signal transduction processes (10). To 
Table I. Assay IDs of human Ran-binding protein 9 variations.

\begin{tabular}{|c|c|c|c|}
\hline Loci & & Probe sequence or Assay-by-design ID* & Method \\
\hline rs24023 & & C_11661329_10 & TaqMan assay \\
\hline rs442458 & & C___580920_10 & TaqMan assay \\
\hline \multirow[t]{4}{*}{ rs6940759 } & Forward & ACCAATGCAGCACAATGATAGGTTA & TaqMan assay by design \\
\hline & Reverse & CAGTAGCAGTAGCAAAGGAGAAGTA & \\
\hline & $\mathrm{VIC}^{\circledast}$ & CTTATTCTGTGACTTTAAAG & \\
\hline & FAM & CTTATTCTGTGATTTTAAAG & \\
\hline rs204226 & & C__ $7615918 \_20$ & TaqMan assay \\
\hline \multirow[t]{4}{*}{ rs16724 } & Forward & GCTATTCCСТCCACCTTTCCTT & TaqMan assay by design \\
\hline & Reverse & TTGAGGAAGTAAAGCTTGAGCTAAGATTT & \\
\hline & $\mathrm{VIC}^{\oplus}$ & CCTGAGTAGGTAGGTCCTA & \\
\hline & FAM & TAGCCTGAGTAGGTCCTA & \\
\hline rs11759518 & & C__ 2974708_10 & TaqMan assay \\
\hline
\end{tabular}

*TaqMan assay IDs from Applied Biosystems, Foster City, CA, USA. FAM, 6-carboxyfluorescein.

date, although the function of RANBP9 is poorly understood, it is considered to be an important regulator of neurite growth through interactions with neural cell adhesion molecules, including L1 (10). It has been suggested that RANBP9, as a ligand for Rho-GEF, may be involved in regulating dendritic spine and branch morphology (11). In addition, RANBP9 has been demonstrated to interact with several important proteins associated with schizophrenia, such as disrupted in schizophrenia 1 (DISC1) and the dopamine D1 receptor in brain tissue $(12,13)$. DISC1 is hypothesized to be associated with major mental illnesses, including schizophrenia, autism and bipolar disorder, and is involved in the regulation of neuronal axon and dendrite outgrowth $(14,15)$. The aberrant dopamine D1 receptor is linked to various neuropsychiatric disorders such as substance abuse, schizophrenia and Parkinson's disease (16). Despite insufficient knowledge with regard to the association between RANBP9 and schizophrenia, RANBP9 is predicted to be involved in the pathogenesis of the disease.

In the present study, a case-control association analysis was conducted between genetic variations in RANBP9 and the risk of schizophrenia and smooth pursuit eye movement (SPEM) abnormalities in a Korean population.

\section{Materials and methods}

Subjects. Trained psychiatrists diagnosed schizophrenia based on the criteria set forth by the Diagnostic and Statistical Manual of Mental Disorders, fourth edition (17). Patients with additional, complicating diagnoses of mental retardation, organic brain damage, drug or alcohol abuse, neurological disorders, autoimmune disorders or low comprehension skills were excluded from the study. Schizophrenia patients $(n=449)$ were recruited over 10 years from Jinju Mental Hospital (Jinju, Korea), Soonyoung Hospital (Sacheon, Korea), Hadong Wooridle Hospital(GyeongsangNamDo,Korea),SeoulNationalUniversity Hospital (Seoul, Korea) and Keyo Hospital (Kyunggi-Do, Korea). Unrelated healthy controls $(n=393)$ were recruited from Hangang Sacred Heart Hospital and the Center for Health
Promotion at Seoul National University Hospital. Peripheral blood leukocytes from 842 samples were collected in tubes containing sodium EDTA and genomic DNA was subsequently obtained using the QIAamp blood extraction kit (Qiagen, Valencia, CA, USA). The study protocols were approved by the institutional review boards of each hospital (Jinju Mental Hospital, Soonyoung Hospital, Hadong Wooridle Hospital, Seoul National University Hospital and Keyo Hospital) and the experiments were conducted with the understanding and written informed consent from each patient.

Measurement of SPEM. In total, 125 schizophrenia patients, who were able to understand the experimental procedure underwent an eye-tracking task as described previously (18). The electrooculographic recordings of the eye movements were used to quantify SPEM abnormality and calculate the natural logarithmic values of the signal/noise $(\mathrm{Ln} \mathrm{S} / \mathrm{N})$ ratio.

Genetic variation selection and genotyping in the RANBP9 gene. In total, five single nucleotide polymorphisms (rs24023, rs442458, rs6940759, rs204226 and rs11759518) and an insertion/deletion (rs16724) variation in RANBP9 were selected from the International HapMap Database (http://hapmap.ncbi. nlm.nih.gov/). Commercially available predesigned TaqMan probes and designed primers (Applied Biosystems, Foster City, CA, USA) were used in genotyping (Table I). All polymorphisms satisfied the following criteria: (i) A minimum call rate of $95 \%$ and (ii) Hardy-Weinberg equilibrium (HWE) of $\mathrm{P}>0.05$. Haplotypes were inferred from the genotyped polymorphisms using the PHASE algorithm, version. 2.0 (19) and those with a frequency of $>0.05$ were included in the association analysis.

Statistical analysis. Lewontin's D' (ID'l) and linkage disequilibrium (LD) coefficient $r^{2}$ between all pairs of biallelic loci using the Haploview algorithm were examined (20). The odds ratio (95\% confidence interval) and corresponding P-values were calculated using a logistic model, controlling for age (continuous value) as a covariate. $\mathrm{P}<0.05$ was considered to 
Table II. Summary of clinical profiles.

\begin{tabular}{|c|c|c|c|}
\hline Parameters & \multicolumn{2}{|c|}{ Schizophrenia patients } & Healthy controls \\
\hline Subjects, n & \multicolumn{2}{|c|}{449} & 393 \\
\hline Average age, years (range) & \multicolumn{2}{|c|}{$44.92(23-76)$} & $54.62(28-80)$ \\
\hline \multirow[t]{2}{*}{ Gender, n (male/female) } & \multicolumn{2}{|c|}{ 250/199 } & $222 / 171$ \\
\hline & Poor $(<3.97)$ & Good ( $(\geq 3.97)$ & \\
\hline Subjects, n & 58 & 67 & \\
\hline Average age, years & $46.0 \pm 9.18$ & $44.2 \pm 9.27$ & \\
\hline Ln S/N ratio* & $3.23 \pm 0.65$ & $4.32 \pm 0.30$ & \\
\hline
\end{tabular}

"Significant difference between poor and good smooth pursuit eye movement groups $(\mathrm{P}<0.001)$. Data are presented as the mean \pm standard error of the mean. Ln S/N, natural logarithmic values of the signal/noise.

Table III. Association analysis of RANBP9 variations with schizophrenia in a Korean population.

\begin{tabular}{|c|c|c|c|c|c|}
\hline \multirow[b]{2}{*}{ Loci } & \multicolumn{2}{|c|}{ MAF } & \multirow[b]{2}{*}{ OR $(95 \% \mathrm{CI})$} & \multirow[b]{2}{*}{ P-value } & \multirow[b]{2}{*}{$\begin{array}{c}\text { Statistical } \\
\text { power }^{\mathrm{a}}\end{array}$} \\
\hline & $\begin{array}{l}\text { Schizophrenia } \\
\quad(n=449)\end{array}$ & $\begin{array}{l}\text { Control } \\
(n=393)\end{array}$ & & & \\
\hline rs24023 & 0.389 & 0.394 & $1.01(0.81-1.26)$ & 0.94 & 95.04 \\
\hline rs442458 & 0.219 & 0.217 & $1.08(0.83-1.41)$ & 0.56 & 87.56 \\
\hline rs6940759 & 0.179 & 0.18 & $0.98(0.74-1.30)$ & 0.89 & 83.04 \\
\hline rs204226 & 0.422 & 0.388 & $1.14(0.91-1.42)$ & 0.25 & 94.97 \\
\hline rs16724 & 0.174 & 0.177 & $0.96(0.72-1.27)$ & 0.76 & 82.58 \\
\hline rs11759518 & 0.089 & 0.087 & $1.04(0.71-1.53)$ & 0.84 & 58.15 \\
\hline ht1 & 0.417 & 0.377 & $1.16(0.93-1.45)$ & 0.19 & 94.81 \\
\hline ht2 & 0.213 & 0.213 & $1.06(0.81-1.38)$ & 0.68 & 87.16 \\
\hline ht3 & 0.177 & 0.213 & $0.77(0.58-1.03)$ & 0.08 & 87.16 \\
\hline ht4 & 0.084 & 0.083 & $1.04(0.70-1.55)$ & 0.86 & 56.40 \\
\hline ht5 & 0.073 & 0.081 & $0.89(0.59-1.34)$ & 0.57 & 55.50 \\
\hline
\end{tabular}

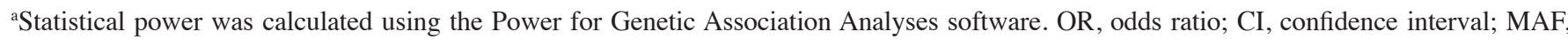
minor allele frequency.

indicated a statistically significant difference between values. The $\mathrm{Ln} \mathrm{S} / \mathrm{N}$ ratio was calculated from analysis of the power spectrum curves and the results were used in logistic analysis of SPEM abnormality, controlling for age as a covariate. Values are presented as the mean \pm standard deviation.

\section{Results}

Characteristics of study subjects. To elucidate whether there is a genetic association between RANBP9 polymorphisms and schizophrenia, a case-control association analysis with 449 schizophrenia patients and 393 healthy subjects was conducted. Table II provides a summary of the clinical profiles in this study. Although the mean age of the control subjects was slightly higher than that of the experimental group, the range of ages was similar in the two groups (Table II). The percentage of males in both groups was also similar (males in case vs. control groups, $55.7 \%$ vs. $56.5 \%$ respectively). Among the case subjects, 125 schizophrenia patients were measured for $\mathrm{Ln} \mathrm{S} / \mathrm{N}$ ratios of SPEM abnormality using an eye-tracking task. The patients were categorized into 'good' and 'poor' performers, according to their SPEM function, based on whether their $\mathrm{Ln} \mathrm{S} / \mathrm{N}$ ratio was above or below 3.97. The $\mathrm{Ln} \mathrm{S} / \mathrm{N}$ ratios (mean \pm standard deviation) of the good and poor SPEM function groups were $4.32 \pm 0.30$ and $3.23 \pm 0.65$, respectively. A significant difference between good and poor groups $(\mathrm{P}<0.001)$ was observed (Table II). Association analysis of SPEM function abnormality in normal controls was not performed due to the low incidence of SPEM impairment in healthy subjects $(<10 \%)$ and association analysis could not retain adequate statistical power due to the low number of samples.

Genotyping of RANBP9 polymorphisms. All RANBP9 polymorphisms were successfully genotyped in a total of 842 subjects; two variations were localized in the 
Table IV. Association analysis of RAN binding protein 9 variations with smooth pursuit eye movement abnormality.

\begin{tabular}{lcccr}
\hline \multicolumn{3}{c}{ MAF } \\
\cline { 2 - 3 } Loci & $\begin{array}{c}\text { Poor } \\
(<3.97, \mathrm{n}=58)\end{array}$ & $\begin{array}{c}\text { Good } \\
(\geq 3.97, \mathrm{n}=67)\end{array}$ & OR $(95 \% \mathrm{CI})$ & P-value \\
\hline rs24023 & 0.439 & 0.368 & $1.28(0.78-2.10)$ & 0.33 \\
rs442458 & 0.239 & 0.193 & $1.14(0.62-2.08)$ & 0.67 \\
rs6940759 & 0.206 & 0.202 & $1.15(0.65-2.04)$ & 0.62 \\
rs204226 & 0.425 & 0.421 & $0.99(0.61-1.61)$ & 0.98 \\
rs16724 & 0.206 & 0.202 & $1.15(0.65-2.04)$ & 0.62 \\
rs11759518 & 0.108 & 0.088 & $1.46(0.60-3.54)$ & 0.41 \\
ht1 & 0.396 & 0.421 & $0.90(0.56-1.46)$ & 0.67 \\
ht2 & 0.209 & 0.193 & $0.98(0.52-1.84)$ & 0.95 \\
ht3 & 0.157 & 0.184 & $0.85(0.44-1.62)$ & 0.62 \\
ht4 & 0.104 & 0.088 & $1.45(0.59-3.54)$ & 0.41 \\
ht5 & 0.075 & 0.088 & $0.91(0.34-2.41)$ & 0.84 \\
\hline
\end{tabular}

OR, odds ratio; CI, confidence interval; MAF, minor allele frequency.

A

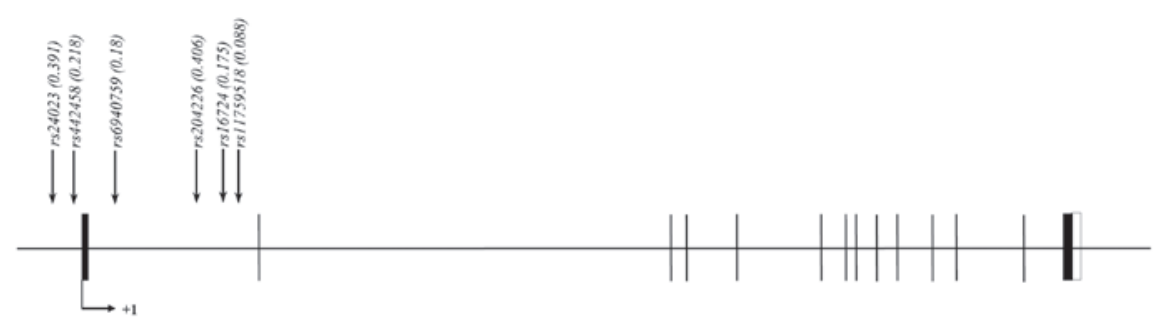

B

C

\begin{tabular}{|c|c|c|c|c|c|c|c|}
\hline Hap. & ญิ ลี & 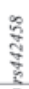 & 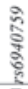 & $\begin{array}{l}\text { ते } \\
\text { కิ } \\
\text { है }\end{array}$ & $\frac{\pi}{6}$ & $\frac{2}{2}$ & Freq. \\
\hline$h t l$ & $\mathrm{~A}$ & c & $\mathrm{T}$ & c & Ins & c & 0.398 \\
\hline$h t 2$ & G & $\mathrm{T}$ & $\mathrm{T}$ & G & Ins & c & 0.213 \\
\hline$h t 3$ & $\mathbf{A}$ & c & $\mathrm{T}$ & G & Ins & c & 0.194 \\
\hline$h t 4$ & G & c & C & G & Del & $\mathrm{T}$ & 0.084 \\
\hline$h t 5$ & G & c & C & G & Del & c & 0.077 \\
\hline$h t 6$ & A & c & C & G & Del & C & 0.011 \\
\hline$h t^{7}$ & G & c & $\mathrm{T}$ & C & Ins & C & 0.007 \\
\hline$h 8$ & G & c & $\mathrm{T}$ & G & Ins & C & 0.006 \\
\hline$h t 9$ & $\mathrm{~A}$ & c & C & G & Del & $\mathrm{T}$ & 0.003 \\
\hline htlo & G & $\mathrm{T}$ & C & G & Del & $\mathrm{T}$ & 0.003 \\
\hline$h t l l$ & G & c & C & G & Ins & C & 0.002 \\
\hline$h t / 2$ & G & $\mathrm{T}$ & $\mathrm{T}$ & c & Ins & c & 0.002 \\
\hline$h t / 3$ & $\mathbf{A}$ & $\mathbf{T}$ & $\mathrm{T}$ & G & Ins & C & 0.001 \\
\hline
\end{tabular}

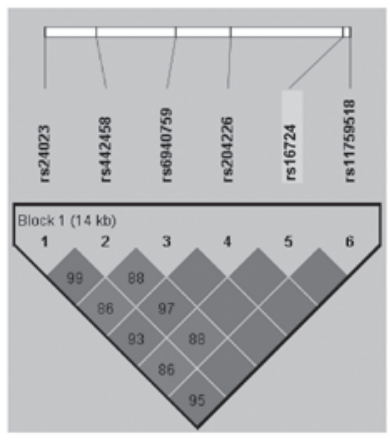

Figure 1: Schematic physical map, haplotypes and LD plot of RANBP9. (A) Physical map of RANBP9. Black boxes indicate an exon region and the white box indicates an UTR. (B) Haplotypes of six RANBP9 polymorphisms. Only the five haplotypes with a frequency of $>0.05$ were used for further analysis. (C) LD plot of six RANBP9 polymorphisms. UTR, untranslated region; LD, linkage disequilibrium; RANBP9, Ran-binding protein 9.

promoter (rs24023 and rs442458) and the other four variants in the intron (rs6940759, rs204226, rs16724 and rs11759518) of the gene (Fig. 1A). No significant deviations from HWE were observed in any of the polymorphisms $(\mathrm{P}>0.05)$. Based on the results of a pair-wise comparison of the variations, a total of 13 haplotypes were inferred (Fig. 1B). Of these, five common haplotypes with frequencies $>0.05$ were identified.

Association analysis of RANBP9 polymorphisms with schizophrenia and SPEM abnormality. Allele frequencies of six polymorphisms were compared between schizophrenia patients and healthy controls using logistic analysis, controlling for age as a covariate. However, no associations between RANBP9 variations and schizophrenia were identified (Table III). In the analysis of SPEM abnormality, no significant association was revealed following the logistic analysis of RANBP9 polymorphisms and haplotypes (Table IV).

\section{Discussion}

RANBP9, also known as RanBPM, was first reported in 2002 as a protein that interacts with the MET proto-oncogene (21). In 
previous studies, RANBP9 was revealed to interact with other important molecules such as amyloid precursor protein (APP) and protein kinase $\mathrm{C}(\mathrm{PKC}) \delta$ and $\gamma(16,22)$. The two kinases have been reported to be associated with mental disorders. Missense mutations in APP have been shown to be associated with cerebral hemorrhage, multi-infarct dementia and Alzheimer's disease, but not directly with schizophrenia (23). However, a recent study has reported the defective metabolism of APP in older schizophrenia patients (age, $>70$ ), suggesting that APP may be involved in schizophrenia etiology (24). PKC is known to phosphorylate the dopamine D1 receptor and negatively regulate dopaminergic signaling (12). A defect in dopamine signaling has been associated with cognitive disorders such as alcohol abuse and schizophrenia and dopamine D1 receptor antagonist SKF-38393 treatment was revealed to improve the cognitive function in animal models with cognitive deficits similar to schizophrenia (25).

Despite the potentially critical role of RANBP9 in mental disorders as previously demonstrated, relatively few studies have been conducted investigating this gene. In a previous study, RANBP9 was observed to be highly expressed in the neurons of the cerebral cortex and the cerebral Purkinje cells in a similar pattern to fragile X mental retardation protein (FMRP) (26). FMRP is known to cause fragile $\mathrm{X}$ syndrome, a common form of hereditary mental retardation. The authors reported that RANBP9 was closely associated with the disorder, and was found to modulate the RNA binding properties of FMRP. In another study, researchers revealed that the concentration of RANBP9-N60, a processed form of RANBP9 almost identical to the RANBP9-Delta1/N60 mutant, was increased in the brain cells of Alzheimer's patients (22). In the study, the authors demonstrated that RANBP9-N60 increased the amyloid $\beta$ generation rate by more than five-fold and therefore drove the amyloid cascade in Alzheimer's disease. The authors hypothesized that the proteolytic processing of RANBP9 may be an ideal therapeutic target.

Although several reports have suggested a possible association between RANBP9 and schizophrenia, the results presented in the current study demonstrated that the genetic variants of RANBP9 were not associated with schizophrenia and SPEM abnormality in a Korean population. However, the present study is the first to conduct association analyses between RANBP9 polymorphisms and schizophrenia. To the best of our knowledge, although, an association study of RANBP9 variants with cognitive disorders such as schizophrenia has not been previously conducted, genetic variants of RANBP1 were recently investigated by our group for possible associations with schizophrenia and SPEM abnormality in a Korean population (27). In the study, two polymorphisms of RANBP1, rs2238798 and rs175162, were genotyped for association analysis. The results indicated that, while the two variations did not exhibit any association with schizophrenia, the haplotype rs2238978G-rs175162T was significantly associated with the risk of SPEM abnormality. According to these results, although RANBP1 and RANBP9 have a similar role, their effects on SPEM abnormality vary. While the exact functions of the proteins are not fully understood, the most prominent difference between the two is that RANBP1 binds specifically with guanosine-5'-triphosphate (GTP)-charged RAN and increases GTP hydrolysis (28).
This difference may account for the association between RANBP1 and SPEM abnormality, however further studies would be required to confirm this hypothesis.

To the best of our knowledge, while the present study is the first to investigate the possible association of RANBP9 variants with schizophrenia and SPEM abnormality, certain limitations are apparent. Due to the rarity of the disease, the number of patients in the study may not have been large enough to generate adequate statistical power. Moreover, the number of schizophrenia patients who were able to test for SPEM was also small. Despite this, the method used to measure SPEM function is used worldwide and is adequate for a genetic association analysis. However, a larger number patient population may be required to confirm the results of the present study. Furthermore, a functional study comparing and contrasting the roles of RANBP1 and RANBP9 may be crucial for deducing the causal variation in SPEM abnormality.

In conclusion, association analyses between RANBP9 variants and the risk of schizophrenia were conducted, however no significant association was identified. Logistic analyses were also conducted to search for a possible association between RANBP9 variants and SPEM abnormality. However, no significant difference was identified. As RANBP9 was only recently discovered, the exact mechanism and function of the protein is not fully understood. Therefore, despite the lack of positive results, the present study may provide a meaningful insight into the role of RANBP9. Further studies comparing a larger number of samples may be beneficial to confirm the results of the present study, as well as elucidate the precise differences between RANBP1 and RANBP9, which may be critical in determining the exact cause of SPEM abnormality.

\section{Acknowledgements}

This study was supported by a grant from the Korea Healthcare Technology R and D Project, Ministry of Health and Welfare, Republic of Korea (grant no. A101023), The National Research Foundation of Korea grant, funded by the Korean government (grant no. 2011-0011935) and the Ministry of Education, Science and Technology, Republic of Korea (grant no. 2009-0093822).

\section{References}

1. McGrath J, Saha S, Chant D and Welham J: Schizophrenia: a concise overview of incidence, prevalence, and mortality. Epidemiol Rev 30: 67-76, 2008.

2. Torrey EF: Prevalence studies in schizophrenia. Br J Psychiatry 150: 598-608, 1987.

3. Park HJ, Westin CF, Kubicki M, et al: White matter hemisphere asymmetries in healthy subjects and in schizophrenia: a diffusion tensor MRI study. Neuroimage 23: 213-223, 2004.

4. Hof PR, Haroutunian V, Friedrich VL, Jr., et al: Loss and altered spatial distribution of oligodendrocytes in the superior frontal gyrus in schizophrenia. Biol Psychiatry 53: 1075-1085, 2003.

5. Hulshoff Pol HE, Schnack HG, Mandl RC, et al: Focal white matter density changes in schizophrenia: reduced inter-hemispheric connectivity. Neuroimage 21: 27-35, 2004.

6. Kubicki M, McCarley RW and Shenton ME: Evidence for white matter abnormalities in schizophrenia. Curr Opin Psychiatry 18: 121-134, 2005.

7. Uranova N, Orlovskaya D, Vikhreva O, et al: Electron microscopy of oligodendroglia in severe mental illness. Brain Res Bull 55: 597-610, 2001. 
8. Uranova NA, Vostrikov VM, Orlovskaya DD and Rachmanova VI: Oligodendroglial density in the prefrontal cortex in schizophrenia and mood disorders: a study from the Stanley Neuropathology Consortium. Schizophr Res 67: 269-275, 2004

9. Mitterauer B: The incoherence hypothesis of schizophrenia: based on decomposed oligodendrocyte-axonic relations. Med Hypotheses 69: 1299-1304, 2007.

10. Murrin LC and Talbot JN: RanBPM, a scaffolding protein in the immune and nervous systems. J Neuroimmune Pharmacol 2 290-295, 2007.

11. Nakayama AY, Harms MB and Luo L: Small GTPases Rac and Rho in the maintenance of dendritic spines and branches in hippocampal pyramidal neurons. J Neurosci 20: 5329-5338, 2000.

12. Rex EB, Rankin ML, Ariano MA and Sibley DR: Ethanol regulation of $\mathrm{D}(1)$ dopamine receptor signaling is mediated by protein kinase $\mathrm{C}$ in an isozyme-specific manner. Neuropsychopharmacology 33: 2900-2911, 2008.

13. Kutay U, Hartmann E, Treichel N, et al: Identification of two novel RanGTP-binding proteins belonging to the importin beta superfamily. J Biol Chem 275: 40163-40168, 2000.

14. Blackwood DH,Fordyce A, Walker MT, St Clair DM,Porteous DJ and Muir WJ: Schizophrenia and affective disorders--cosegregation with a translocation at chromosome 1q42 that directly disrupts brain-expressed genes: clinical and P300 findings in a family. Am J Hum Genet 69: 428-433, 2001.

15. Millar JK, Christie S, Anderson S, et al: Genomic structure and localisation within a linkage hotspot of Disrupted In Schizophrenia 1, a gene disrupted by a translocation segregating with schizophrenia. Mol Psychiatry 6: 173-178, 2001.

16. Rex EB, Rankin ML, Yang Y, et al: Identification of RanBP $9 / 10$ as interacting partners for protein kinase C (PKC) gamma/delta and the D1 dopamine receptor: regulation of PKC-mediated receptor phosphorylation. Mol Pharmacol 78: 69-80, 2010

17. American Psychiatric Association: Diagnostic and Statistical Manual of Mental Disorders. 4th edition. American Psychiatric Association, Washington, D.C., WA, 1994.
18. Park BL, Shin HD, Cheong HS, et al: Association analysis of COMT polymorphisms with schizophrenia and smooth pursuit eye movement abnormality. J Hum Genet 54: 709-712, 2009.

19. Stephens M, Smith NJ and Donnelly P: A new statistical method for haplotype reconstruction from population data. Am J Hum Genet 68: 978-989, 2001.

20. Barrett JC, Fry B, Maller J and Daly MJ: Haploview: analysis and visualization of LD and haplotype maps. Bioinformatics 21: 263-265, 2005

21. Wang D, Li Z, Messing EM and Wu G: Activation of Ras/Erk pathway by a novel MET-interacting protein RanBPM. J Biol Chem 277: 36216-36222, 2002.

22. Lakshmana MK, Hayes CD, Bennett SP, et al: Role of RanBP9 on amyloidogenic processing of APP and synaptic protein levels in the mouse brain. FASEB J 26: 2072-2083, 2012.

23. Morris S, Leung J, Sharp C, Blackwood D, Muir W and St Clair D: Screening schizophrenic patients for mutations in the amyloid precursor protein gene. Psychiatr Genet 4: 23-27, 1994.

24. Albertini V, Benussi L, Paterlini A, et al: Distinct cerebrospinal fluid amyloid-beta peptide signatures in cognitive decline associated with Alzheimer's disease and schizophrenia. Electrophoresis 33: 3738-3744, 2012.

25. McLean SL, Idris NF, Woolley ML and Neill JC: D(1)-like receptor activation improves PCP-induced cognitive deficits in animal models: Implications for mechanisms of improved cognitive function in schizophrenia. Eur Neuropsychopharmacol 19: 440-450, 2009.

26. Menon RP, Gibson TJ and Pastore A: The C terminus of fragile $\mathrm{X}$ mental retardation protein interacts with the multi-domain Ran-binding protein in the microtubule-organising centre. J Mol Biol 343: 43-53, 2004.

27. Cheong HS, Park BL, Kim EM, et al: Association of RANBP1 haplotype with smooth pursuit eye movement abnormality. Am J Med Genet B Neuropsychiatr Genet 156B: 67-71, 2011.

28. Kehlenbach RH, Dickmanns A, Kehlenbach A, Guan T and Gerace L: A role for RanBP1 in the release of CRM1 from the nuclear pore complex in a terminal step of nuclear export. J Cell Biol 145: 645-657, 1999. 